\title{
Gauge independent quantum gravitational corrections to Maxwell's equation
}

\section{S. Katuwal and R.P. Woodard}

Department of Physics, University of Florida, Gainesville, FL 32611, U.S.A.

E-mail: sanjib.katuwal@ufl.edu, woodard@phys.ufl.edu

ABSTRACT: We consider quantum gravitational corrections to Maxwell's equations on flat space background. Although the vacuum polarization is highly gauge dependent, we explicitly show that this gauge dependence is canceled by contributions from the source which disturbs the effective field and the observer who measures it. Our final result is a gauge independent, real and causal effective field equation that can be used in the same way as the classical Maxwell equation.

KEYwords: Effective Field Theories, Models of Quantum Gravity, Renormalization Regularization and Renormalons

ArXiv EPrint: 2107.13341 


\section{Contents}

1 Introduction 1

2 Including the source and the observer $\quad 3$

2.1 Correlation between vertices 5

2.2 Vertex-force carrier correlations 6

$\begin{array}{lll}2.3 & \text { Vertex-source and vertex-observer correlations } & 7\end{array}$

2.4 Source-observer correlations 9

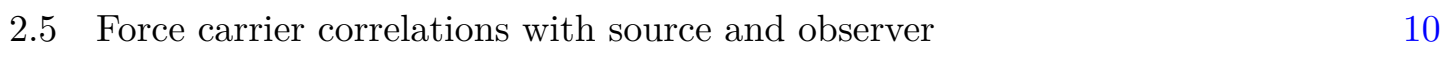

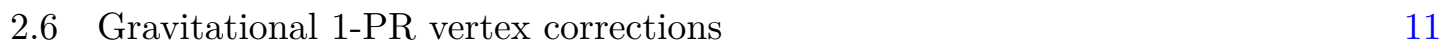

$\begin{array}{lll}2.7 & \text { Sum total } & 12\end{array}$

$\begin{array}{lll}3 & \text { Conclusions } & 13\end{array}$

$\begin{array}{ll}\text { A The vertices } & 14\end{array}$

$\begin{array}{ll}\text { B Propagators } & 15\end{array}$

$\begin{array}{lr}\text { C The Donoghue Identities } & 16\end{array}$

\section{Introduction}

The greatest story ever told in physics is how a century of brilliant experimental extemporization culminated in the development of Maxwell's equations. This was humanity's first relativistic, unified field theory and it set the stage for the discoveries of general relativity and non-Abelian gauge theories. Electrodynamics is still one of the core subjects in the study of physics. Most western physicists recall the ingenuity and perseverance required of them as graduate students to solve Maxwell's equations in the wide variety of settings treated in the classic text by the late J.D. Jackson [1].

Quantum loop corrections to electrodynamics are small at low frequencies, and those from quantum gravity are unobservable. One might therefore expect that including these effects causes only a small change in electrodynamics. The math is simple enough: one first computes the 1PI (one-particle-irreducible) 2-photon function, $\left.i^{[\mu} \Pi^{\nu}\right]\left(x ; x^{\prime}\right)$, known as the "vacuum polarization". Then Maxwell's equations are supplemented by the integral of the vacuum polarization contracted into the vector potential $A_{\nu}\left(x^{\prime}\right)$,

$$
\partial_{\nu} F^{\nu \mu}(x)+\int d^{4} x^{\prime}\left[{ }^{\mu} \Pi^{\nu}\right]\left(x ; x^{\prime}\right) A_{\nu}\left(x^{\prime}\right)=J^{\mu}(x),
$$

where $F_{\mu \nu} \equiv \partial_{\mu} A_{\nu}-\partial_{\nu} A_{\mu}$ is the field strength tensor and $J^{\mu}(x)$ is the current density. However, students of quantum field theory are strongly enjoined that they cannot think of 
solving the quantum-corrected equation the same as its classical analog; they must instead abandon the concept of local fields and infer physics entirely from scattering amplitudes. Although basing physics on scattering amplitudes is valid for most situations on flat space background, it does seem to be an over-reaction, and it is not even possible in cosmology. The purpose of this paper is to provide a version of the quantum-corrected field equation (1.1) which can be solved as in classical electrodynamics.

Part of the reason for the curious dichotomy between classical and quantum is the prevalence of the "in-out" formalism so elegantly summarized by the Feynman rules. The in-out vacuum polarization is neither real, nor is it causal in the sense of vanishing for points $x^{\prime \mu}$ outside the past light-cone of $x^{\mu}$. Those two properties are not errors; in-out amplitudes are precisely the right objects of study for computing scattering amplitudes. However, the absence of reality and causality is certainly problematic if one wishes to regard solutions to the quantum-corrected field equation (1.1) as electric and magnetic fields.

Julian Schwinger long ago devised a method for computing true expectation values which is almost as simple to use as the Feynman rules [2]. When the vacuum polarization of the Schwinger-Keldysh formalism is employed in equation (1.1) the effective field equations become manifestly real and causal [3-10]. However, there is still an obstacle: the propagators of vector and tensor fields require gauge fixing, and loop corrections involving these propagators cause the vacuum polarization to depend on the choice of gauge. For example, single graviton loop corrections to the vacuum polarization on a $D$-dimensional flat space background $\left(g_{\mu \nu} \equiv \eta_{\mu \nu}+\kappa h_{\mu \nu}\right.$ with $\left.\kappa^{2}=16 \pi G\right)$ with the most general, Poincaré invariant gauge fixing functional,

$$
\mathcal{L}_{G F}=-\frac{1}{2 a} \eta^{\mu \nu} F_{\mu} F_{\nu}, \quad F_{\mu}=\eta^{\rho \sigma}\left(h_{\mu \rho, \sigma}-\frac{b}{2} h_{\rho \sigma, \mu}\right),
$$

result in a primitive vacuum polarization of the form [11],

$$
i\left[{ }^{\mu} \Pi^{\nu}\right]\left(x ; x^{\prime}\right)=-\frac{\kappa^{2} \mathcal{C}_{0}(D, a, b)(D-2) \Gamma^{2}\left(\frac{D}{2}-1\right)}{32(D-1) \pi^{D}}\left(\eta^{\mu \nu} \partial^{2}-\partial^{\mu} \partial^{\nu}\right) \frac{1}{\left(x-x^{\prime}\right)^{2 D-2}},
$$

where the gauge dependent multiplicative factor is,

$$
\begin{aligned}
\mathcal{C}_{0}(D, a, b)= & D(D-2)(D-3)+\frac{(D-1)(D-2)^{2}\left[(D-2)(a-1)-D(b-1)^{2}\right]}{2(b-2)^{2}} \\
& +(D-1)(D-2)^{2}(D-4)\left[-\frac{(a-1)}{2}+\frac{2}{D-2}\left(\frac{b-1}{b-2}\right)\right] .
\end{aligned}
$$

Although the tensor structure and spacetime dependence of (1.3) is universal, the multiplicative factor $\mathcal{C}(D, a, b)$ can be made to range from $-\infty$ to $+\infty$ by adjusting the gauge parameters $a$ and $b[11]$.

John Donoghue has shown how to use general relativity as a low energy effective field theory to reliably compute quantum gravitational corrections to the long-range potentials induced by the exchange of massless particles such as photons and gravitons [12, 13]. His technique is to compute the scattering amplitude between two massive particles which interact with the massless field, and then use inverse scattering theory to infer the exchange 
potential. In this way one can derive gauge independent, single graviton loop corrections to the Newtonian potential $[14,15]$ and to the Coulomb potential [16].

It has recently been noted that Donoghue's S-matrix technique can be short-circuited to produce gauge independent effective field equations directly, without passing through the intermediate stages of computing scattering amplitudes and solving the inverse scattering problem [17]. The key is applying position space versions of a series of identities derived by Donoghue and collaborators for the purpose of isolating the nonlocal and nonanalytic parts of scattering amplitudes which correct long-range potentials [13, 18]. These identities degenerate the massive propagators of the particles being scattered to delta functions, thus casting the important parts of higher-point contributions to 2-particle scattering in a form that can be regarded as corrections to the 1PI 2-point function of the massless field. In this picture the gauge dependence of the original effective field equation derives from having omitted to include quantum gravitational interactions with the source which disturbs the effective field and from the observer who measures it; and the corrections to the 1PI 2-point function repair this omission. The new technique has already been implemented at one loop order for quantum gravitational corrections to a massless scalar on flat space background, and its independence of the gauge parameters $a$ and $b$ explicitly demonstrated [17]. In this paper we do the same for quantum gravitational corrections to electrodynamics, which is a realistic system and one involving vector fields.

This paper closely follows the analysis of Bjerrum-Bohr [16] who applied Donoghue's technique to include one graviton loop corrections to electrodynamics on flat space background. Section 2 goes through the position-space version of each of the same diagrams he considered, including first order perturbations of the gauge parameters (1.2),

$$
\left\{\begin{array}{l}
a \equiv 1+\delta a \\
b \equiv 1+\delta b
\end{array}\right\} \quad \Longrightarrow \quad \mathcal{C}_{0}(4, a, b)=8+12 \cdot \delta a+0 \cdot \delta b+O\left(\delta^{2}\right)
$$

In each case we show how the Donoghue identities allow one to regard the diagram as a correction to the vacuum polarization. Of course the gauge dependence cancels when everything is summed up, and the result has the same form (1.3), but with the constant $\mathcal{C}_{0}(4, a, b)$ replaced by the gauge independent number +40 . Our conclusions comprise section 3. Three appendices give, respectively, the vertices, the propagators and the Donoghue identities, including the new one we required for certain of the $\delta b$ contributions.

\section{Including the source and the observer}

In this section, we use the scattering of a pair of massive, charged scalars to provide the source which disturbs the effective field and the observer who measures this disturbance. The Lagrangian that describes the scattering is,

$$
\mathcal{L}=\left[\frac{R}{16 \pi G}-\frac{1}{4} g^{\alpha \mu} g^{\beta \nu} F_{\alpha \nu} F_{\mu \beta}-\left(D_{\mu} \phi\right)^{*} g^{\mu \nu}\left(D_{\nu} \phi\right)+m^{2} \phi^{*} \phi\right] \sqrt{-g},
$$

where, $D_{\mu}=\nabla_{\mu}-i e A_{\mu}$ and $\nabla_{\mu}$ denotes the metric-compatible covariant derivative. (When acting on a scalar the covariant derivative degenerates to the partial derivative, 


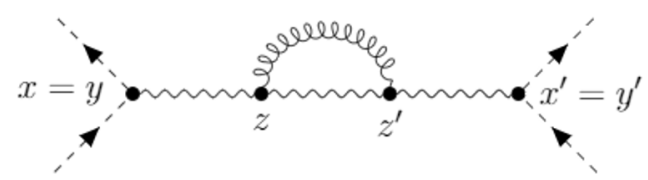

Figure 1. This diagram shows how the vacuum polarization contributes to the amputated 4-scalar vertex function. Dashed lines represent massive scalars, wavy lines represent photons and curly lines represent gravitons. These graphs have the same topology as Bjerrum-Bohr's Diagram 8 [16].

$\left.D_{\mu} \phi=\partial_{\mu} \phi-i e A_{\mu} \phi.\right)$ Unless otherwise stated, we work with the usual $c=\hbar=1$ convention of particle physics, however, we employ a spacelike metric. General relativity plus SQED (Scalar Quantum Electrodynamics) is treated as a low energy effective field theory in the sense of Donoghue $[12,13]$. The perturbation is around flat space with the following definitions of the graviton field $h_{\mu \nu}$ and the loop counting parameter $\kappa^{2}$,

$$
g_{\mu \nu}(x) \equiv \eta_{\mu \nu}+\kappa h_{\mu \nu}, \quad \kappa^{2} \equiv 16 \pi G .
$$

The vertices we require are listed in appendix A, and the various propagators are given in appendix B.

Our procedure for purging gauge dependence from the one loop vacuum polarization is to write down position space representations for each of the order $e^{2} \kappa^{2}$ contributions to the amputated 4-scalar function. Any external derivatives are assumed to act on the external scalar wave functions appropriate to 2-particle scattering. By exploiting the various Donoghue Identities of appendix $\mathrm{C}$ to degenerate the (internal) massive scalar propagators to Dirac delta functions, we reduce each contribution to a form that can be interpreted as a correction to $i\left[{ }^{\mu} \Pi^{\nu}\right]\left(x ; x^{\prime}\right)$.

We begin by considering the contribution of the original, gauge dependent vacuum polarization to the amputated 4-scalar function as shown in figure 1. The expression for this diagram is

$$
\begin{aligned}
i V_{0}\left(x ; x^{\prime}\right)= & e\left(\partial_{x} \downarrow-\partial_{x} \uparrow\right)^{\alpha} \times e\left(\partial_{x^{\prime}} \downarrow-\partial_{x^{\prime}} \uparrow\right)^{\beta} \\
& \times \int d^{D} z i\left[{ }_{\alpha} \Delta_{\mu}\right](x ; z) \int d^{D} z^{\prime} i\left[{ }_{\beta} \Delta_{\nu}\right]\left(x^{\prime} ; z^{\prime}\right) \times i\left[{ }^{\mu} \Pi^{\nu}\right]\left(z ; z^{\prime}\right)
\end{aligned}
$$

where the vacuum polarization was given in (1.3) and external derivatives with an up (down) arrow act on upper (lower) scalar wave functions at that vertex. First note that Poincaré invariance and partial integration allows us to act all longitudinal parts on the external legs, where (by current conservation) they vanish due to the on-shell condition,

$$
(\partial \downarrow-\partial \uparrow)_{\mu}(\partial \downarrow+\partial \uparrow)^{\mu}=\left(\partial^{2} \downarrow-m^{2}\right)-\left(\partial^{2} \uparrow-m^{2}\right) .
$$

We can also use the relation,

$$
\frac{1}{\Delta x^{2 D-2}}=\frac{\partial^{2}}{2(D-2)^{2}} \frac{1}{\Delta x^{2 D-4}}=\frac{\partial^{2}}{2(D-2)^{2}}\left[\frac{4 \pi^{\frac{D}{2}}}{\Gamma\left(\frac{D}{2}-1\right)} i \Delta\left(x ; x^{\prime}\right)\right]^{2},
$$




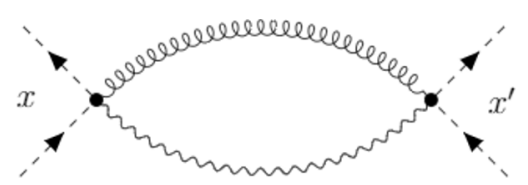

Figure 2. This diagram shows the contribution of graviton correlation between two vertices. Dashed lines represent massive scalars, wavy lines represent photons and curly lines represent gravitons. These graphs have the same topology as Bjerrum-Bohr's Diagram 4 [16].

to attain the form,

$$
\begin{aligned}
i V_{0}\left(x ; x^{\prime}\right)= & -\frac{e^{2} \kappa^{2} \mathcal{C}_{0}(D, a, b)}{4(D-1)(D-2)} \times\left(\partial_{x} \downarrow-\partial_{x} \uparrow\right)_{\mu}\left(\partial_{x^{\prime}} \downarrow-\partial_{x^{\prime}} \uparrow\right)^{\mu} \\
& \times \int d^{D} z i \Delta(x ; z) \int d^{D} z^{\prime} i \Delta\left(x^{\prime} ; z^{\prime}\right) \partial_{z}^{2} \partial_{z^{\prime}}^{2}\left[i \Delta\left(z ; z^{\prime}\right)\right]^{2} .
\end{aligned}
$$

The final step is to partially integrate the factors of $\partial_{z}^{2}$ and $\partial_{z^{\prime}}^{2}$ to act on the massless propagators, and use the delta functions that result from the propagator equation (B.1) to eliminate the integrations over $z^{\mu}$ and $z^{\prime \mu}$,

$$
i V_{0}\left(x ; x^{\prime}\right)=\frac{\mathcal{C}_{0}(D, a, b)}{(D-1)(D-2)} \times \frac{e^{2} \kappa^{2}}{4}\left(\partial_{x} \downarrow-\partial_{x} \uparrow\right) \cdot\left(\partial_{x^{\prime}} \downarrow-\partial_{x^{\prime}} \uparrow\right) \times\left[i \Delta\left(x ; x^{\prime}\right)\right]^{2} .
$$

After applying the appropriate Donoghue Identity from appendix $\mathrm{C}$ it turns out that all contributions to the amputated 4-scalar function take this same form, with different gauge dependent multiplicative factors. To simplify the notation, we define a new gauge dependent constant which includes the factor of $1 /(D-1)(D-2)$, and we take $D=4$ because dimensional regularization plays no role, while also dropping higher order perturbations in the gauge parameters $a=1+\delta a$ and $b=1+\delta b$,

$$
\frac{\mathcal{C}_{0}(D, a, b)}{(D-1)(D-2)} \equiv C_{0}(\delta a, \delta b)+O\left(D-4, \delta^{2}\right) .
$$

In other words, $C_{0}(\delta a, \delta b)=\frac{4}{3}+2 \delta a$.

\subsection{Correlation between vertices}

The correlation between source $\left(\right.$ at $x^{\prime \mu}$ ) and observer (at $\left.x^{\mu}\right)$ vertices is the first extra contribution to the amputated 4-scalar function, as shown in figure 2. This diagram corresponds to the analytic expression,

$$
\begin{aligned}
i V_{1}\left(x ; x^{\prime}\right)= & \frac{1}{2} e \kappa\left[\eta^{\delta \mu} \eta^{\nu \alpha}+\eta^{\delta \nu} \eta^{\mu \alpha}-\eta^{\mu \nu} \eta^{\delta \alpha}\right]\left(\partial_{x} \uparrow-\partial_{x} \downarrow\right)_{\alpha} \\
& \times \frac{1}{2} e \kappa\left[\eta^{\gamma \rho} \eta^{\sigma \beta}+\eta^{\sigma \gamma} \eta^{\rho \beta}-\eta^{\rho \sigma} \eta^{\gamma \beta}\right]\left(\partial_{x^{\prime}} \uparrow-\partial_{x^{\prime}} \downarrow\right)_{\beta} \\
& \times i\left[{ }_{\mu \nu} \Delta_{\rho \sigma}\right]\left(x ; x^{\prime}\right) \times i\left[{ }_{\gamma} \Delta_{\delta}\right]\left(x ; x^{\prime}\right) .
\end{aligned}
$$

Substituting the appropriate propagators from appendix B, contracting all the indices, simplifying and making use of the relation,

$$
i \Delta\left(x ; x^{\prime}\right) \frac{\partial_{\mu} \partial_{\nu}}{\partial^{2}} i \Delta\left(x ; x^{\prime}\right)=\frac{1}{4} \times \frac{\Gamma^{2}\left(\frac{D}{2}-1\right)}{16 \pi^{D}}\left[\frac{\eta_{\mu \nu}}{\Delta x^{2 D-4}}+\frac{\partial_{\mu} \partial_{\nu}}{(2 D-6)} \frac{1}{\Delta x^{2 D-6}}\right],
$$



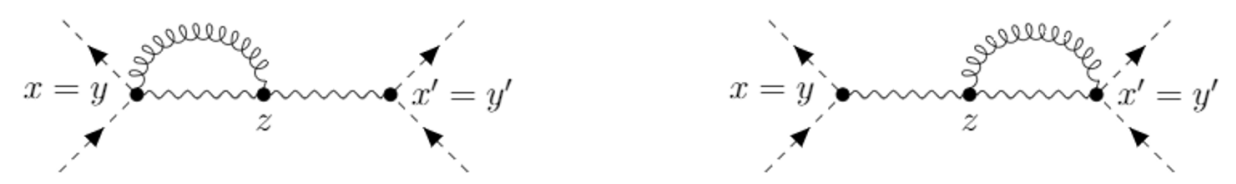

Figure 3. These diagrams show the contributions from correlations between the force carrier and one of the vertices. Dashed lines represent massive scalars, wavy lines represent photons and curly lines represent gravitons. These graphs have the same topology as Bjerrum-Bohr's Diagram 7 [16].

gives,

$$
i V_{1}\left(x ; x^{\prime}\right)=\frac{e^{2} \kappa^{2} \Gamma^{2}\left(\frac{D}{2}-1\right)}{16 \pi^{D}}\left[D+\frac{(3 D-2) \delta a}{4}-\frac{(D-2)^{2} \delta b}{4}\right]\left(\partial_{x} \downarrow-\partial_{x} \uparrow\right)_{\mu}\left(\partial_{x^{\prime}} \downarrow-\partial_{x^{\prime}} \uparrow\right)_{\nu} \frac{\eta_{\mu \nu}}{\Delta x^{2 D-4}} .
$$

Note that the second term in the square bracket of expression (2.10) drops out by current conservation.

Recognizing the massless scalar propagator (B.2) provides a simpler form for (2.11),

$$
i V_{1}\left(x ; x^{\prime}\right)=\left[D+\frac{(3 D-2) \delta a}{4}-\frac{(D-2)^{2} \delta b}{4}\right] \times e^{2} \kappa^{2}\left(\partial_{x} \downarrow-\partial_{x} \uparrow\right) \cdot\left(\partial_{x^{\prime}} \downarrow-\partial_{x^{\prime}} \uparrow\right) \times\left[i \Delta\left(x ; x^{\prime}\right)\right]^{2} .
$$

As promised, expression (2.11) takes the same form as the vacuum polarization contribution (2.7), but with a different gauge dependent, multiplicative constant. By comparison with (2.7) we can recognize,

$$
\mathcal{C}_{1}(D, a, b)=4(D-1)(D-2)\left[D+\frac{(3 D-2) \delta a}{4}-\frac{(D-2)^{2} \delta b}{4}+O\left(\delta^{2}\right)\right] .
$$

Henceforth we will not bother with dimensional regularization, and we will make the same notational simplification as (2.8). This means that the vertex-vertex correction is,

$$
i V_{1}\left(x ; x^{\prime}\right)=C_{1}(\delta a, \delta b) \times \frac{e^{2} \kappa^{2}}{4}\left(\partial_{x} \downarrow-\partial_{x} \uparrow\right) \cdot\left(\partial_{x^{\prime}} \downarrow-\partial_{x^{\prime}} \uparrow\right) \times\left[i \Delta\left(x ; x^{\prime}\right)\right]^{2},
$$

where $C_{1}(\delta a, \delta b)=16+10 \delta a-4 \delta b$.

\subsection{Vertex-force carrier correlations}

The next contribution comes from the correlations between a single vertex and the exchange photon, as shown in figure 3. The analytic form is,

$$
\begin{aligned}
i V_{2}\left(x ; x^{\prime}\right)= & \frac{1}{2} e \kappa\left[\eta^{\epsilon \mu} \eta^{\nu \beta}+\eta^{\epsilon \nu} \eta^{\mu \beta}-\eta^{\mu \nu} \eta^{\epsilon \beta}\right]\left(\partial_{x} \uparrow-\partial_{x} \downarrow\right)_{\beta} \times e\left(\partial_{x^{\prime}} \downarrow-\partial_{x^{\prime}} \uparrow\right)^{\theta} \\
& \times \int d^{D} z\left(-i \kappa V^{\gamma \delta \alpha \tau \rho \sigma}\right) \partial_{z \tau} i\left[{ }_{\epsilon} \Delta_{\delta}\right](x ; z) \partial_{z \alpha} i\left[{ }_{\gamma} \Delta_{\theta}\right]\left(z ; x^{\prime}\right) \times i\left[{ }_{\mu \nu} \Delta_{\rho \sigma}\right](x ; z) \\
& + \text { (Permutation) }
\end{aligned}
$$



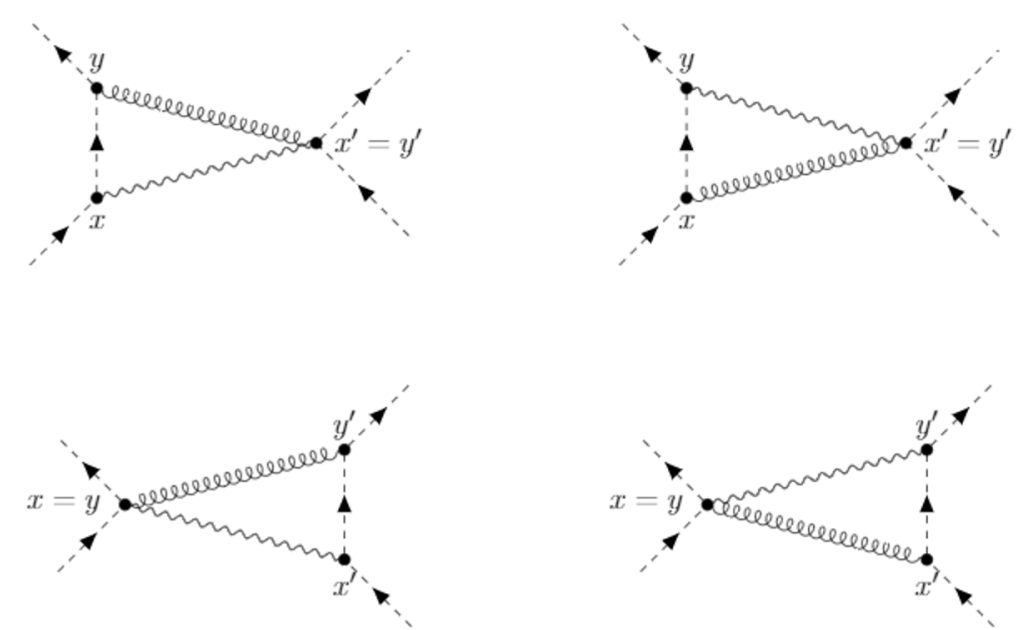

Figure 4. These diagrams show the contributions from correlations between the source (primed) or observer (unprimed) and the opposite vertex. Dashed lines represent massive scalars, wavy lines represent photons and curly lines represent gravitons. These graphs have the same topology as Bjerrum-Bohr's Diagram 3 [16].

For reducing this diagram it is useful to note how the product of a massless propagator times one of the gauge variations can be expressed as a differential operator acting on a single function of the Poincaré interval,

$$
\begin{aligned}
i \Delta\left(x ; x^{\prime}\right) \frac{\partial_{\mu} \partial_{\nu}}{\partial^{2}} i \Delta\left(x ; x^{\prime}\right)= & \frac{1}{4} \eta_{\mu \nu}\left[i \Delta\left(x ; x^{\prime}\right)\right]^{2}-\frac{1}{8} \partial_{\mu} \partial_{\nu} I\left\{\left[i \Delta\left(x ; x^{\prime}\right)\right]^{2}\right\}, \\
\partial_{\kappa} i \Delta\left(x ; x^{\prime}\right) \frac{\partial_{\alpha} \partial_{\beta}}{\partial^{2}} i \Delta\left(x ; x^{\prime}\right)= & \frac{D-2}{16(D-1)} \partial_{\kappa} \partial_{\alpha} \partial_{\beta} I\left\{\left[i \Delta\left(x ; x^{\prime}\right)\right]^{2}\right\}+\frac{D}{8(D-1)} \eta_{\alpha \beta} \partial_{\kappa}\left[i \Delta\left(x ; x^{\prime}\right)\right]^{2} \\
& -\frac{D-2}{8(D-1)}\left(\eta_{\kappa \alpha} \partial_{\beta}+\eta_{\kappa \beta} \partial_{\alpha}\right)\left[i \Delta\left(x ; x^{\prime}\right)\right]^{2},
\end{aligned}
$$

where the symbol $I\{\}$ represents indefinite integration of the argument with respect to $\Delta x^{2}$. The final result for these diagrams is,

$$
i V_{2}\left(x ; x^{\prime}\right)=C_{2}(\delta a, \delta b) \times \frac{e^{2} \kappa^{2}}{4}\left(\partial_{x} \downarrow-\partial_{x} \uparrow\right) \cdot\left(\partial_{x^{\prime}} \downarrow-\partial_{x^{\prime}} \uparrow\right) \times\left[i \Delta\left(x ; x^{\prime}\right)\right]^{2},
$$

where $C_{2}(\delta a, \delta b)=-12-16 \delta a+4 \delta b$.

\subsection{Vertex-source and vertex-observer correlations}

We next consider contribution from correlations between the source, or observer, and the opposite vertex, as shown in figure 4. (Correlations with nearer vertices do not contribute because they are cancelled by field strength renormalization.) We use $x^{\mu}\left(y^{\mu}\right)$ for incoming (outgoing) observer, and $x^{\prime \mu}\left(y^{\prime \mu}\right)$ for incoming (outgoing) source. We also adopt the notation that a bar over a vertex with only a single external leg denotes differentiation of the on-shell external wave function. With these conventions we can write the analytic form 
of the diagrams in figure 4 as,

$$
\begin{aligned}
\text { Figure } 4= & \frac{i \kappa}{2}\left[\bar{\partial}_{y}^{\mu} \partial_{y}^{\nu}+\bar{\partial}_{y}^{\nu} \partial_{y}^{\mu}-\eta^{\mu \nu}\left(\partial_{y} \cdot \bar{\partial}_{y}+m^{2}\right)\right] \times e\left(\bar{\partial}_{x}-\partial_{x}\right)^{\delta} i \Delta_{m}(x ; y) \\
& \left.\times \frac{e \kappa}{2}\left[\eta^{\gamma \rho} \eta^{\alpha \sigma}+\eta^{\gamma \sigma} \eta^{\alpha \rho}-\eta^{\rho \sigma} \eta^{\alpha \gamma}\right]\left(\partial_{x^{\prime}} \uparrow-\partial_{x^{\prime}} \downarrow\right)_{\alpha} \times i{ }_{\delta} \Delta_{\gamma}\right]\left(x ; x^{\prime}\right) \times i\left[{ }_{\mu \nu} \Delta_{\rho \sigma}\right]\left(y ; x^{\prime}\right) \\
& +(3 \text { permutations }) .
\end{aligned}
$$

As can be seen from figure 4, these contributions involve an internal massive scalar propagator in the loop. This poses an obstacle to regarding expression (2.19) as a correction to the vacuum polarization. This is overcome through the "Donoghue Identities" of appendix $\mathrm{C}$, which degenerate the massive scalar propagator to a Dirac delta function, and reduce expression (2.19) to the same 2-point form (2.7) as the contribution from the original vacuum polarization. The part of expression (2.19) which is independent of the gauge parameters $\delta a$ and $\delta b$ reaches the desired form through the Donoghue Identities (C.1) and (C.2).

As an example of the part of (2.19) proportional to $\delta a$, we consider the term,

$$
\begin{aligned}
\delta a & \times \frac{i \kappa}{2}\left[\bar{\partial}_{y}^{\mu} \partial_{y}^{\nu}+\bar{\partial}_{y}^{\nu} \partial_{y}^{\mu}-\eta^{\mu \nu}\left(\partial_{y} \cdot \bar{\partial}_{y}+m^{2}\right)\right] \times e\left(\bar{\partial}_{x}-\partial_{x}\right)^{\delta} i \Delta_{m}(x ; y) \\
& \times \frac{e \kappa}{2}\left[\eta^{\gamma \rho} \eta^{\alpha \sigma}+\eta^{\gamma \sigma} \eta^{\alpha \rho}-\eta^{\rho \sigma} \eta^{\alpha \gamma}\right]\left(\partial_{x^{\prime}} \uparrow-\partial_{x^{\prime}} \downarrow\right)_{\alpha} \times i\left[{ }_{\delta} \Delta_{\gamma}\right]\left(x ; x^{\prime}\right) \times \eta_{\nu \rho} \frac{\partial_{\mu} \partial_{\sigma}}{\partial^{2}} i \Delta\left(y ; x^{\prime}\right) .
\end{aligned}
$$

The derivative $\partial_{\mu}$ acting on the massless propagator $i \Delta\left(y ; x^{\prime}\right)$ can be partially integrated to act on the massive propagator $i \Delta_{m}(x ; y)$,

$$
-\left(\bar{\partial}_{y}+\partial_{y}\right)_{\mu}\left[\bar{\partial}_{y}^{\mu} \partial_{y}^{\nu}+\bar{\partial}_{y}^{\nu} \partial_{y}^{\mu}-\eta^{\mu \nu}\left(\partial_{y} \cdot \bar{\partial}_{y}+m^{2}\right)\right]=-\bar{\partial}_{y}^{\nu} i \delta^{D}(x-y) .
$$

The remaining factor of $\partial^{\sigma} / \partial^{2}$ can be reduced using,

$$
\begin{aligned}
i \Delta\left(x ; x^{\prime}\right) \times \frac{\partial_{\alpha}}{\partial^{2}} i \Delta\left(x ; x^{\prime}\right) & =\frac{1}{4} \partial_{\alpha} I\left\{\left[i \Delta\left(x ; x^{\prime}\right)\right]^{2}\right\}, \\
\partial^{2} I\left\{\left[i \Delta\left(x ; x^{\prime}\right)\right]^{2}\right\} & \longrightarrow-2(D-4)\left[i \Delta\left(x ; x^{\prime}\right)\right]^{2} .
\end{aligned}
$$

From expression (B.8) for the graviton propagator we see that there are two parts proportional to $\delta b$. The second term with $\partial_{\mu} \partial_{\nu} / \partial^{2}$ can be reduced just like (2.20). The other term requires additional effort,

$$
\begin{aligned}
-2 \delta b & \times \frac{i \kappa}{2}\left[\bar{\partial}_{y}^{\mu} \partial_{y}^{\nu}+\bar{\partial}_{y}^{\nu} \partial_{y}^{\mu}-\eta^{\mu \nu}\left(\partial_{y} \cdot \bar{\partial}_{y}+m^{2}\right)\right] \times e\left(\bar{\partial}_{x}-\partial_{x}\right)^{\delta} i \Delta_{m}(x ; y) \\
& \times \frac{e \kappa}{2}\left[\eta^{\gamma \rho} \eta^{\alpha \sigma}+\eta^{\gamma \sigma} \eta^{\alpha \rho}-\eta^{\rho \sigma} \eta^{\alpha \gamma}\right]\left(\partial_{x^{\prime}} \uparrow-\partial_{x^{\prime}} \downarrow\right)_{\alpha} \times i\left[{ }_{\delta} \Delta_{\gamma}\right]\left(x ; x^{\prime}\right) \times \eta_{\mu \nu} \frac{\partial_{\rho} \partial_{\sigma}}{\partial^{2}} i \Delta\left(y ; x^{\prime}\right) .
\end{aligned}
$$

To reduce this term we distinguish between $y^{\mu}$ derivatives acting on the external leg $\left(\bar{\partial}_{y}^{\mu}\right)$, the massive propagator $\left(\partial_{y}^{\mu}\right)$ and the massless propagator of the graviton $\left(\tilde{\partial}_{y}^{\mu}\right)$,

$$
\bar{\partial}_{y}^{\mu}+\partial_{y}^{\mu}+\tilde{\partial}_{y}^{\mu}=0 \text {. }
$$

Now note that,

$$
\eta_{\mu \nu}\left[\bar{\partial}_{y}^{\mu} \partial_{y}^{\nu}+\bar{\partial}_{y}^{\nu} \partial_{y}^{\mu}-\eta^{\mu \nu}\left(\partial_{y} \cdot \bar{\partial}_{y}+m^{2}\right)\right]=\left(\bar{\partial}_{y}^{2}-m^{2}\right)+\left(\partial_{y}^{2}-m^{2}\right)-\tilde{\partial}_{y}^{2}-2 m^{2} .
$$



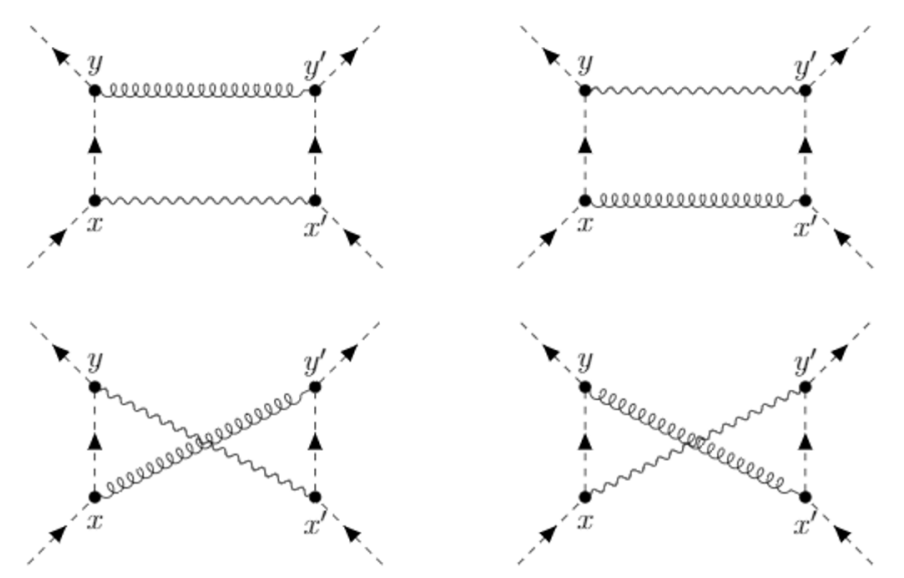

Figure 5. These diagrams show the contributions from correlations between the source (primed) and observer (unprimed). Dashed lines represent massive scalars, wavy lines represent photons and curly lines represent gravitons. These graphs have the same topology as Bjerrum-Bohr's Diagram $2[16]$.

The factor of $\left(\bar{\partial}_{y}^{2}-m^{2}\right)$ vanishes due to the external leg being on shell. The next term in (2.26) degenerates the massive scalar propagator,

$$
\left(\partial_{y}^{2}-m^{2}\right) i \Delta_{m}(x ; y)=i \delta^{D}(x-y)
$$

Of course the factor of $\tilde{\partial}_{y}^{2}$ eliminates the troublesome inverse D'Alembertian, whereupon the Donoghue Identity (C.3) completes the reduction. The final term in (2.26) requires the new Donoghue Identities (C.5) and (C.6).

Putting everything together gives the final result for figure 4,

$$
i V_{3}\left(x ; x^{\prime}\right)=C_{3}(\delta a, \delta b) \times \frac{e^{2} \kappa^{2}}{4}\left(\partial_{x} \downarrow-\partial_{x} \uparrow\right) \cdot\left(\partial_{x^{\prime}} \downarrow-\partial_{x^{\prime}} \uparrow\right) \times\left[i \Delta\left(x ; x^{\prime}\right)\right]^{2},
$$

where $C_{3}(\delta a, \delta b)=-32-8 \delta a-2 \delta b$.

\subsection{Source-observer correlations}

Figure 5 shows contributions from correlations between source and observer. (Correlations between the source and itself, or the observer and itself, do not correct the exchange photon.) The analytic form for these diagrams is,

$$
\begin{aligned}
\text { (Figure 5) }= & \frac{i \kappa}{2}\left[\bar{\partial}_{y}^{\mu} \partial_{y}^{\nu}+\bar{\partial}_{y}^{\nu} \partial_{y}^{\mu}-\eta^{\mu \nu}\left(\bar{\partial}_{y} \cdot \partial_{y}+m^{2}\right)\right]\left(\bar{\partial}_{x}-\partial_{x}\right)^{\delta} i \Delta_{m}(x ; y) \\
& \times \frac{i \kappa}{2}\left[\bar{\partial}_{y^{\prime}}^{\rho} \partial_{y^{\prime}}^{\sigma}+\bar{\partial}_{y^{\prime}}^{\sigma} \partial_{y^{\prime}}^{\rho}-\eta^{\rho \sigma}\left(\bar{\partial}_{y^{\prime}} \cdot \partial_{y^{\prime}}+m^{2}\right)\right]\left(\bar{\partial}_{x^{\prime}}-\partial_{x^{\prime}}\right)^{\gamma} i \Delta_{m}\left(x^{\prime} ; y^{\prime}\right) \\
& \times i\left[{ }_{\gamma} \Delta_{\delta}\right]\left(x ; x^{\prime}\right) \times i\left[{ }_{\mu \nu} \Delta_{\rho \sigma}\right]\left(y ; y^{\prime}\right)+(3 \text { Permutations })
\end{aligned}
$$

Note that the two permutations on the bottom line of figure 5 contain an extra minus sign due to the 2-scalar-1-photon vertex. 

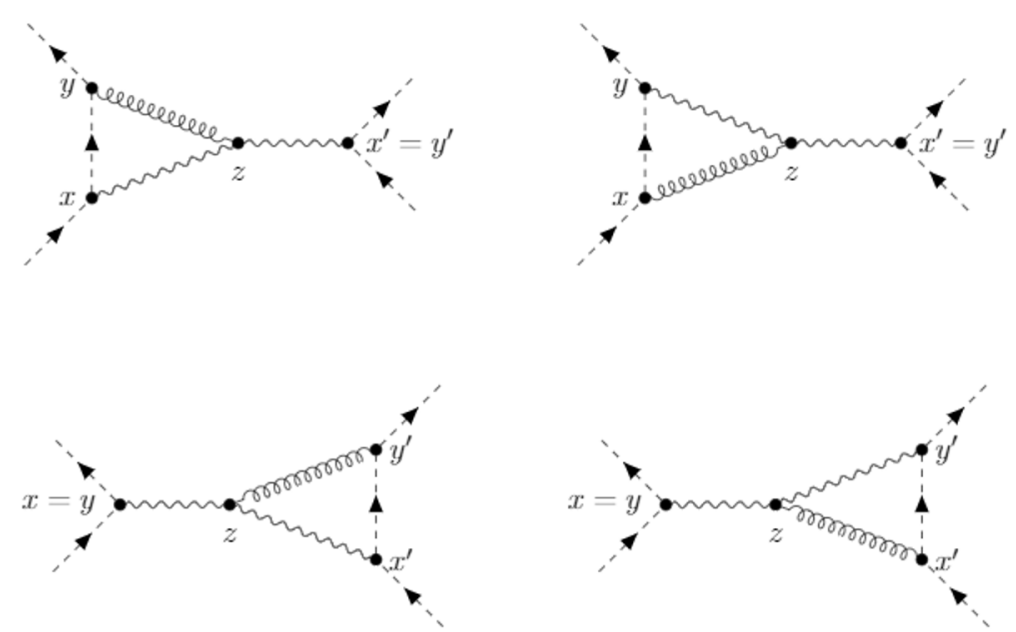

Figure 6. These diagrams show the contributions from correlations between the source (primed) or observer (unprimed) and the force carrier. Dashed lines represent massive scalars, wavy lines represent photons and curly lines represent gravitons. These graphs have the same topology as Bjerrum-Bohr's Diagram 6 [16].

Reducing the part of (2.29) independent of $\delta a$ and $\delta b$ is accomplished by the Donoghue Identity (C.4). The reduction of the gauge dependent parts is similar to what we have seen before with one difference: after using relation (2.21), one must combine parts from the various diagrams to eliminate some troublesome terms. The final result for figure 5 is,

$$
i V_{4}\left(x ; x^{\prime}\right)=C_{4}(\delta a, \delta b) \times \frac{e^{2} \kappa^{2}}{4}\left(\partial_{x} \downarrow-\partial_{x} \uparrow\right) \cdot\left(\partial_{x^{\prime}} \downarrow-\partial_{x^{\prime}} \uparrow\right) \times\left[i \Delta\left(x ; x^{\prime}\right)\right]^{2},
$$

where $C_{4}(\delta a, \delta b)=\frac{80}{3}+0 \cdot \delta a+2 \delta b$.

\subsection{Force carrier correlations with source and observer}

The next contribution comes from correlations between the source or observer and the photon. The Feynman diagrams are given in figure 6, and the analytic expression is,

$$
\begin{aligned}
\text { (Figure 6) }= & \frac{i \kappa}{2}\left[\bar{\partial}_{y}^{\mu} \partial_{y}^{\nu}+\bar{\partial}_{y}^{\nu} \partial_{y}^{\mu}-\eta^{\mu \nu}\left(\bar{\partial}_{y} \cdot \partial_{y}+m^{2}\right)\right]\left(\bar{\partial}_{x}-\partial_{x}\right)^{\epsilon} i \Delta_{m}(x ; y) \\
& \times \int d^{D} z\left(-i \kappa V^{\gamma \delta \alpha \tau \rho \sigma}\right) \frac{\partial}{\partial z^{\tau}} i\left[{ }_{\epsilon} \Delta_{\delta}\right](x ; z) \frac{\partial}{\partial z^{\alpha}} i\left[{ }_{\gamma} \Delta_{\theta}\right]\left(z ; x^{\prime}\right) \times e\left(\partial_{x^{\prime}} \downarrow-\partial_{x^{\prime}} \uparrow\right)^{\theta} \\
& \times i\left[{ }_{\mu \nu} \Delta_{\rho \sigma}\right]+(3 \text { Permutations }) .
\end{aligned}
$$

The reduction process is almost same as in section 2.3, the chief difference being the extra photon propagator. We extract a D'Alembertian and then use (B.1) to eliminate this extra propagator and the integration over $z^{\mu}$. The final contribution from these diagrams is,

$$
i V_{5}\left(x ; x^{\prime}\right)=C_{5}(\delta a, \delta b) \times \frac{e^{2} \kappa^{2}}{4}\left(\partial_{x} \downarrow-\partial_{x} \uparrow\right) \cdot\left(\partial_{x^{\prime}} \downarrow-\partial_{x^{\prime}} \uparrow\right) \times\left[i \Delta\left(x ; x^{\prime}\right)\right]^{2},
$$

where $C_{5}(\delta a, \delta b)=12+12 \delta a+4 \delta b$. 

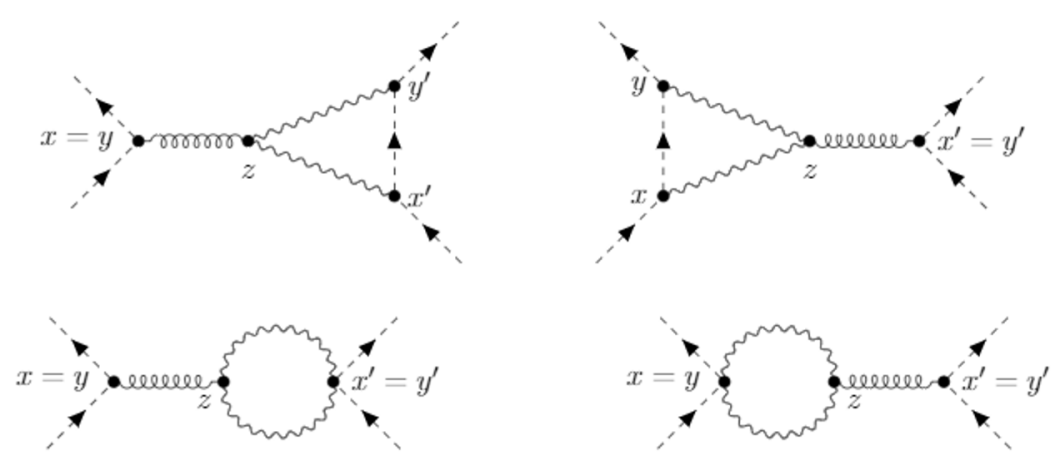

Figure 7. These diagrams show the contributions from the 1PR (one particle reducible) diagrams corresponding to gravitational vertex corrections. Dashed lines represent massive scalars, wavy lines represent photons and curly lines represent gravitons. These graphs have the same topology as Bjerrum-Bohr's Diagram 5 [16].

\subsection{Gravitational 1-PR vertex corrections}

The final contribution to the amputated 4-scalar function comes from diagrams in which a loop of photons corrects one of the vertices and the graviton carries the exchange force. The relevant diagrams are shown in figure 7. The analytic form for first two diagrams is,

Figure $7_{\text {up }}=\frac{i \kappa}{2}\left[\partial_{x}^{\mu \uparrow} \partial_{x}^{\nu} \downarrow+\partial_{x}^{\nu} \uparrow \partial_{x}^{\mu} \downarrow-\eta^{\mu \nu}\left(\partial_{x} \uparrow \cdot \partial_{x} \downarrow+m^{2}\right)\right] \times e\left(\bar{\partial}_{x^{\prime}}-\partial_{x^{\prime}}\right)^{\theta}$

$$
\begin{aligned}
& \times e\left(\partial_{y^{\prime}}-\bar{\partial}_{y^{\prime}}\right)^{\epsilon} i \Delta_{m}\left(x^{\prime} ; y^{\prime}\right) \int d^{D} z\left(-i \kappa V^{\gamma \delta \alpha \tau \rho \sigma}\right) \frac{\partial}{\partial z^{\tau}} i\left[{ }_{\epsilon} \Delta_{\delta}\right]\left(z ; x^{\prime}\right) \frac{\partial}{\partial z^{\alpha}} i\left[{ }_{\gamma} \Delta_{\theta}\right]\left(z ; x^{\prime}\right) \\
& \times i\left[{ }_{\mu \nu} \Delta_{\rho \sigma}\right](x ; z)+\text { (Permutation). }
\end{aligned}
$$

The second two diagrams are,

$$
\begin{aligned}
\text { Figure } 7_{\text {down }}= & \frac{1}{2} \times \frac{i \kappa}{2}\left[\partial_{x}^{\mu \uparrow} \partial_{x}^{\nu} \downarrow+\partial_{x}^{\nu \uparrow} \partial_{x}^{\mu} \downarrow-\eta^{\mu \nu}\left(\partial_{x} \uparrow \partial_{x} \downarrow+m^{2}\right)\right] \times\left(-2 i e^{2} \eta^{\epsilon \theta}\right) \\
& \int d^{D} z\left(-i \kappa V^{\gamma \delta \alpha \tau \rho \sigma}\right) \frac{\partial}{\partial z^{\tau}} i\left[{ }_{\epsilon} \Delta_{\delta}\right]\left(z ; y^{\prime}\right) \frac{\partial}{\partial z^{\alpha}} i\left[{ }_{\gamma} \Delta_{\theta}\right]\left(z ; x^{\prime}\right) \\
& \times i\left[{ }_{\mu \nu} \Delta_{\rho \sigma}\right](x ; z)+(\text { Permutation })
\end{aligned}
$$

Whenever possible, it is prudent to partially integrate and reflect a derivative through the graviton propagator to act on the 2-scalar-1-graviton vertex. One then makes use of the relation,

$$
\left(\partial_{x} \downarrow+\partial_{x} \uparrow\right)_{\mu}\left[\partial_{x}^{\mu} \uparrow \partial_{x}^{\nu} \downarrow+\partial_{x}^{\nu} \uparrow \partial_{x}^{\mu} \downarrow-\eta^{\mu \nu}\left(\partial_{x} \uparrow \partial_{x} \downarrow+m^{2}\right)\right]=0 .
$$

The rest of the reduction is same as in earlier sections. The final result is,

$$
i V_{6}\left(x ; x^{\prime}\right)=C_{6}(\delta a, \delta b) \times \frac{e^{2} \kappa^{2}}{4}\left(\partial_{x} \downarrow-\partial_{x} \uparrow\right) \cdot\left(\partial_{x^{\prime}} \downarrow-\partial_{x^{\prime}} \uparrow\right) \times\left[i \Delta\left(x ; x^{\prime}\right)\right]^{2},
$$

where $C_{6}(\delta a, \delta b)=-\frac{16}{3}+0 \cdot \delta a-4 \delta b$. 


\begin{tabular}{|c|c|c|c|c|}
\hline$i$ & Description & $O_{i}$ & $A_{i}$ & $B_{i}$ \\
\hline 0 & Vacuum Polarization & $+\frac{4}{3}$ & +2 & 0 \\
\hline 1 & Circular Diagram & +16 & +10 & -4 \\
\hline 2 & Vertex-Force Carrier & -12 & -16 & +4 \\
\hline 3 & Triangular Diagrams & -32 & -8 & -2 \\
\hline 4 & Box Diagrams & $+\frac{80}{3}$ & 0 & +2 \\
\hline 5 & Source, Obs.- Force Carrier & +12 & +12 & +4 \\
\hline 6 & Graviton 1PR Vertex & $-\frac{16}{3}$ & 0 & -4 \\
\hline & Total & $+\frac{20}{3}$ & 0 & 0 \\
\hline
\end{tabular}

Table 1. The entry on the $i^{\text {th }}$ row represent the gauge dependent factors for the contribution coming from the diagram in figure $i+1$.

\subsection{Sum total}

As we have explained, the Donoghue Identities of appendix C allow us to cast each contribution to the amputated 4-scalar function in the form,

$$
i V_{i}\left(x ; x^{\prime}\right)=C_{i}(\delta a, \delta b) \times \frac{e^{2} \kappa^{2}}{4}\left(\partial_{x} \downarrow-\partial_{x} \uparrow\right) \cdot\left(\partial_{x^{\prime}} \downarrow-\partial_{x^{\prime}} \uparrow\right) \times\left[i \Delta\left(x ; x^{\prime}\right)\right]^{2},
$$

where the gauge dependent constant is $C_{i}(\delta a, \delta b)=O_{i}+A_{i} \delta a+B_{i} \delta b$. Table 1 summarizes our results. Of course the point of the exercise is to total the $O_{i}$ 's, and to show that the terms proportional to $\delta a$ and $\delta b$ sum to zero,

$$
\sum_{i=0}^{6} i V_{i}\left(x ; x^{\prime}\right)=+\frac{20}{3} \times \frac{e^{2} \kappa^{2}}{4}\left(\partial_{x} \downarrow-\partial_{x} \uparrow\right) \cdot\left(\partial_{x^{\prime}} \downarrow-\partial_{x^{\prime}} \uparrow\right) \times\left[i \Delta\left(x ; x^{\prime}\right)\right]^{2} .
$$

We then reverse the steps that led from expression (2.3) to (2.7) in order to conclude that the gauge independent vacuum polarization from a single graviton loop is,

$$
i\left[{ }^{\mu} \Pi^{\nu}\right]\left(x ; x^{\prime}\right)=-\frac{40}{3} \times \frac{\kappa^{2}}{16 \pi^{4}}\left[\eta^{\mu \nu} \partial^{2}-\partial^{\mu} \partial^{\nu}\right] \frac{1}{\Delta x^{2 D-2}} .
$$




\section{Conclusions}

The main result of this paper is that including quantum gravitational corrections from the source which disturbs the effective field, and from the observer who measures the disturbance, eliminates the massive gauge dependence of the quantum-corrected Maxwell equation (1.1) that was evident in the multiplicative constant $\mathcal{C}_{0}(D, a, b)$ of expression (1.4). After renormalization, and application of the Schwinger-Keldysh formalism [10], our final result for the one loop effective field equation is,

$$
\partial_{\nu} F^{\nu \mu}(x)+\frac{5 \hbar G \partial^{6}}{48 \pi^{2} c^{3}} \int d^{4} x^{\prime} \theta(\Delta t-\Delta r)\left\{\ln \left[\mu^{2}\left(\Delta t^{2}-r^{2}\right)\right]-1\right\} \partial_{\nu}^{\prime} F^{\nu \mu}\left(x^{\prime}\right)=J^{\mu}(x),
$$

where $\Delta t \equiv t-t^{\prime}, r \equiv\left\|\vec{x}-\vec{x}^{\prime}\right\|$ and we have restored the factors $\hbar$ and $c$. Although equation (3.1) is not local, it is real and causal.

For a static point charge $J^{\mu}(t, \vec{x})=q \delta^{\mu}{ }_{0} \delta^{3}\left(\vec{x}-\vec{x}^{\prime}\right)$ the quantum-corrected Coulomb potential is,

$$
\Phi(r)=\frac{q}{4 \pi r}\left\{1+\frac{10 \hbar G}{3 \pi r^{2} c^{3}}+\mathcal{O}\left(G^{2}\right)\right\} .
$$

This result agreees with Bjerrum-Bohr [16], but we now have the ability to solve for quantum gravitational corrections to the full range of problems one encounters in classical electrodynamics. These corrections are bound to be quite small under ordinary conditions, although the potential for slightly super-luminal propagation is noteworthy [11], and was predicted long ago $[19,20]$.

Although it is nice to finally be able to include quantum gravitational corrections to Maxwell's equations on flat space background, we could always have inferred physics from scattering amplitudes. The real necessity for our method is for studying quantum gravitational corrections to electrodynamics in cosmology. These effects can be significant, especially during the epoch of primordial inflation. For example, when the simplest de Sitter background gauge [21, 22] is employed to compute single graviton loop corrections to the vacuum polarization [23] one finds corrections to the Coulomb potential [24], and to the photon field strength [25] which become nonperturbatively strong at large distances and late times. When the vacuum polarization is computed in a much more complicated, 1-parameter family of gauges [26], one finds the same time dependence for the photon field strength, but with a different numerical coefficient [27], signaling a slight gauge dependence which must be eliminated to infer reliable results. First order corrections to the graviton propagator in the de Sitter generalization of the gauge (1.2) have been derived recently [28]. This should facilitate extending the current work to de Sitter background.

\section{Acknowledgments}

We are grateful to J.F. Donoghue for correspondence on this subject. This work was partially supported by NSF grant PHY-1912484 and by the Institute for Fundamental Theory at the University of Florida. 


\section{A The vertices}

- 2-Scalars-1-Photon Vertex

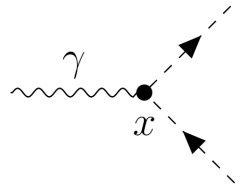

-2-Scalars-2-Photon Vertex

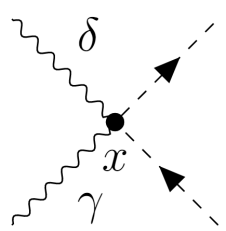

-2-Scalars-1-Graviton Vertex

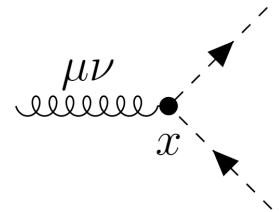

- 2-Scalars-1-Photon-1-Graviton Vertex

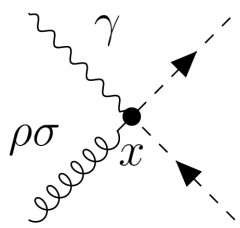

- 2-Photon-1-Graviton Vertex

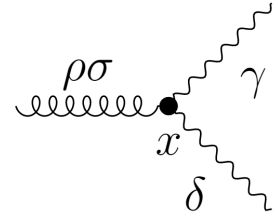

$$
=e\left(\partial_{x} \downarrow-\partial_{x} \uparrow\right)^{\gamma}
$$

$$
=-2 i e^{2} \eta^{\gamma \delta}
$$

$$
\begin{gathered}
=\frac{i \kappa}{2}\left[\partial_{x}^{\mu} \uparrow \partial_{x}^{\nu} \downarrow+\partial_{x}^{\nu} \uparrow \partial_{x}^{\mu} \downarrow\right. \\
\left.-\eta^{\mu \nu}\left(\partial_{x} \uparrow \cdot \partial_{x} \downarrow+m^{2}\right)\right]
\end{gathered}
$$

$$
\begin{gathered}
=\frac{e \kappa}{2}\left[\eta^{\gamma \rho} \eta^{\alpha \sigma}+\eta^{\gamma \sigma} \eta^{\alpha \rho}-\eta^{\rho \sigma} \eta^{\alpha \gamma}\right] \\
\times\left(\partial_{x} \uparrow-\partial_{x} \downarrow\right)_{\alpha}
\end{gathered}
$$

$$
\begin{aligned}
& =-i \kappa V^{\gamma \delta \alpha \tau \rho \sigma} \partial_{\alpha} \uparrow \partial_{\tau} \downarrow \\
& \text { where, } \\
& V^{\gamma \delta \alpha \tau \rho \sigma}=\eta^{\rho \sigma} \eta^{\alpha[\tau} \eta^{\delta] \gamma}+4 \eta^{\rho)[\gamma} \eta^{\alpha][\delta} \eta^{\tau](\sigma}
\end{aligned}
$$




\section{B Propagators}

The massless scalar propagator $i \Delta\left(x ; x^{\prime}\right)$ obeys the equation,

$$
\partial^{2} i \Delta\left(x ; x^{\prime}\right)=i \delta^{D}\left(x-x^{\prime}\right) .
$$

Even in $D$ spacetime dimensions it has a simple expression in terms of the Lorentz interval $\Delta x^{2}\left(x ; x^{\prime}\right)$

$$
i \Delta\left(x ; x^{\prime}\right)=\frac{\Gamma\left(\frac{D}{2}-1\right)}{4 \pi^{D / 2}}\left(\frac{1}{\Delta x^{2}}\right)^{\frac{D}{2}-1},
$$

where we define,

$$
\Delta x^{2}\left(x ; x^{\prime}\right) \equiv\left\|\vec{x}-\vec{x}^{\prime}\right\|^{2}-\left(\left|t-t^{\prime}\right|-i \varepsilon\right)^{2} .
$$

The massive scalar propagator $i \Delta_{m}\left(x ; x^{\prime}\right)$ obeys the equation,

$$
\left(\partial^{2}-m^{2}\right) i \Delta_{m}\left(x ; x^{\prime}\right)=i \delta^{D}\left(x-x^{\prime}\right) .
$$

It can be written in terms of Bessel functions but the expression itself is not necessary for our purposes. It turns out that we can always eliminate $i \Delta_{m}\left(x ; x^{\prime}\right)$, either with the propagator equation (B.4) or by recourse to one of the Donoghue Identities given in appendix C.

The photon field also requires gauge fixing. The most general Poincaré invariant gauge fixing functional depends upon an arbitrary parameter $c$,

$$
\mathcal{L}_{\text {EMfix }}=-\frac{1}{2 c}\left(\partial^{\mu} A_{\mu}\right)^{2} .
$$

The associated propagator can be expressed using the massless scalar propagator (B.2),

$$
i\left[{ }_{\rho} \Delta_{\sigma}\right]\left(x ; x^{\prime}\right)=\left[\eta_{\rho \sigma}+(c-1) \frac{\partial_{\rho} \partial_{\sigma}}{\partial^{2}}\right] i \Delta\left(x ; x^{\prime}\right) .
$$

The longitudinal term proportional to $c-1$ presumably drops out due to current conservation but we shall simply adopt the $c=1$ Feynman gauge that Bjerrum-Bohr employed [16],

$$
i\left[{ }_{\rho} \Delta_{\sigma}\right]\left(x ; x^{\prime}\right)=\eta_{\rho \sigma} i \Delta\left(x ; x^{\prime}\right) .
$$

The most general Poincaré invariant gauge fixing function (1.2) depends on two parameters $a$ and $b \neq 2$ (for $b=2$ the gauge fixing functional degenerates to the square of a linearized Ricci scalar). To simplify the analysis we work only to first order in the perturbations $a=1+\delta a$ and $b=1+\delta b$,

$$
\begin{aligned}
i\left[{ }_{\mu \nu} \Delta_{\rho \sigma}\right]\left(x ; x^{\prime}\right)=\left[2 \eta_{\mu(\rho} \eta_{\sigma) \nu}-\frac{2 \eta_{\mu \nu} \eta_{\rho \sigma}}{D-2}+\frac{4 \delta a \partial_{(\mu} \eta_{\nu)(\rho} \partial_{\sigma)}}{\partial^{2}}\right. & \\
& \left.-2 \delta b\left(\eta_{\mu \nu} \frac{\partial_{\rho} \partial_{\sigma}}{\partial^{2}}+\eta_{\rho \sigma} \frac{\partial_{\mu} \partial_{\nu}}{\partial^{2}}\right)\right] i \Delta\left(x ; x^{\prime}\right) .
\end{aligned}
$$




\section{The Donoghue Identities}

What we term the "Donoghue Identities" are not equalities but rather relations for extracting the nonlocal and nonanalytic parts of amplitudes which can contribute to long range forces. As originally derived by Donoghue and collaborators [12, 13, 18], they included nonlinear classical effects as well as quantum effects, but we have retained only the parts relevant for quantum effects. When expressed in position space these relations all have the effect of degenerating massive propagators to delta functions. We required six such relations, of which the final two (those involving factors of $1 / \partial^{2}$ ) were derived by us for this project:

- This concerns 3-point diagrams with no derivatives acting on propagators,

$$
i \Delta_{m}(x ; y) i \Delta\left(x ; x^{\prime}\right) i \Delta\left(y ; x^{\prime}\right) \longrightarrow \frac{i \delta^{D}(x-y)}{2 m^{2}}\left[i \Delta\left(x ; x^{\prime}\right)\right]^{2} .
$$

- This concerns 3-point diagrams with a derivative acting on a massless propagator,

$$
\left[\partial_{x}^{\mu} i \Delta\left(x ; x^{\prime}\right)\right] i \Delta_{m}(x ; y) i \Delta\left(y ; x^{\prime}\right) \longrightarrow-\partial_{x}^{\mu}\left[\frac{i \delta^{D}(x-y)}{2 m^{2}}\left[i \Delta\left(x ; x^{\prime}\right)\right]^{2}\right] .
$$

- This concerns 3-point diagrams with two derivatives acting on a massless propagator,

$$
\begin{aligned}
{\left[\partial_{x}^{\mu} \partial_{x}^{\nu} i \Delta\left(x ; x^{\prime}\right)\right] i \Delta_{m}(x ; y) } & \Delta\left(y ; x^{\prime}\right) \rightarrow\left\{\partial_{x}^{\mu} \partial_{x}^{\nu} \frac{\left(\partial_{x}+\partial_{y}\right)^{2}}{2 m^{2}}-\frac{1}{2}\left(\partial_{x}^{\mu}\left(\partial_{x}+\partial_{y}\right)^{\nu}\right.\right. \\
& \left.\left.+\partial_{x}^{\nu}\left(\partial_{x}+\partial_{y}\right)^{\mu}\right)-\frac{1}{4} \eta^{\mu \nu}\left(\partial_{x}+\partial_{y}\right)^{2}\right\}\left[\frac{i \delta^{D}(x-y)}{2 m^{2}}\left[i \Delta\left(x ; x^{\prime}\right)\right]^{2}\right]
\end{aligned}
$$

- These concern 4-point diagrams with no derivatives acting on the propagators. The first is relevant to the box diagrams as shown on the upper part of figure 5. The second is relevant to the cross diagrams as shown on the lower part of figure 5,

$$
\begin{aligned}
m^{2}\left(\partial_{x}+\partial_{y}\right)^{2} & {\left[i \Delta_{m}(x ; y) i \Delta\left(y ; y^{\prime}\right) i \Delta_{m}\left(y^{\prime} ; x^{\prime}\right) i \Delta\left(x^{\prime} ; x\right)\right] } \\
& \longrightarrow\left(1-\frac{\partial_{x} \cdot \partial_{x^{\prime}}-m^{2}}{3 m^{2}}\right)\left[i \Delta\left(x ; x^{\prime}\right)\right]^{2} \delta^{D}(x-y) \delta^{D}\left(x^{\prime}-y^{\prime}\right) \\
m^{2}\left(\partial_{x}+\partial_{y}\right)^{2} & {\left[i \Delta_{m}(x ; y) i \Delta\left(y ; x^{\prime}\right) i \Delta_{m}\left(x^{\prime} ; y^{\prime}\right) i \Delta\left(y^{\prime} ; x\right)\right] } \\
& \longrightarrow\left(-1+\frac{\partial_{x} \cdot \partial_{y^{\prime}}-m^{2}}{3 m^{2}}\right)\left[i \Delta\left(x ; x^{\prime}\right)\right]^{2} \delta^{D}(x-y) \delta^{D}\left(x^{\prime}-y^{\prime}\right)
\end{aligned}
$$

- This concerns 3-point diagrams with a derivative and an inverse Laplacian on a massless propagator,

$$
\begin{aligned}
& i \Delta_{m}(x ; y) i \Delta\left(x ; x^{\prime}\right) \frac{\partial^{\mu}}{\partial^{2}} \Delta\left(y ; x^{\prime}\right) \\
& \quad \longrightarrow \frac{1}{m^{2}}\left[\frac{\left(\partial_{x}+\partial_{y}\right)^{\mu}}{8}-\frac{\partial_{x}^{m u}}{2}+\frac{1}{3 m^{2}} \partial_{x}^{\mu}\left(\partial_{x}+\partial_{y}\right)^{2}\right]\left[\frac{i \delta^{D}(x-y)}{2 m^{2}}\left[i \Delta\left(x ; x^{\prime}\right)\right]^{2}\right] .
\end{aligned}
$$


- This concerns 3-point diagrams with two derivatives and an inverse Laplacian on a massless propagator,

$$
\begin{aligned}
i \Delta_{m}(x ; y) i \Delta\left(x ; x^{\prime}\right) \frac{\partial^{\mu} \partial^{\nu}}{\partial^{2}} \Delta\left(y ; x^{\prime}\right) & \\
\longrightarrow & {\left[\frac{1}{2} \eta^{\mu \nu}-\frac{1}{m^{2}} \partial_{x}^{\mu} \partial_{x}^{\nu}+\frac{1}{6 m^{2}} \eta_{\mu \nu}\left(\partial_{x}+\partial_{y}\right)^{2}+\frac{1}{2 m^{2}}\left(\partial_{x}^{\mu}\left(\partial_{x}+\partial_{y}\right)^{\nu}\right.\right.} \\
& \left.\left.+\partial_{x}^{\nu}\left(\partial_{x}+\partial_{y}\right)^{\mu}\right)-\frac{2}{3 m^{4}}\left(\partial_{x}+\partial_{y}\right)^{2} \partial_{x}^{\mu} \partial_{x}^{\nu}\right]\left[\frac{i \delta^{D}(x-y)}{2 m^{2}}\left[i \Delta\left(x ; x^{\prime}\right)\right]^{2}\right] .
\end{aligned}
$$

Open Access. This article is distributed under the terms of the Creative Commons Attribution License (CC-BY 4.0), which permits any use, distribution and reproduction in any medium, provided the original author(s) and source are credited.

\section{References}

[1] J.D. Jackson, Classical Electrodynamics, Wiley \& Sons, New York (1998) [INSPIRE].

[2] J.S. Schwinger, Brownian motion of a quantum oscillator, J. Math. Phys. 2 (1961) 407 [INSPIRE].

[3] K.T. Mahanthappa, Multiple production of photons in quantum electrodynamics, Phys. Rev. 126 (1962) 329 [INSPIRE].

[4] P.M. Bakshi and K.T. Mahanthappa, Expectation value formalism in quantum field theory. 1, J. Math. Phys. 4 (1963) 1 [inSPIRE].

[5] P.M. Bakshi and K.T. Mahanthappa, Expectation value formalism in quantum field theory. 2, J. Math. Phys. 4 (1963) 12 [inSPIRE].

[6] L.V. Keldysh, Diagram technique for nonequilibrium processes, Zh. Eksp. Teor. Fiz. 47 (1964) 1515 [INSPIRE].

[7] K.-c. Chou, Z.-b. Su, B.-l. Hao and L. Yu, Equilibrium and Nonequilibrium Formalisms Made Unified, Phys. Rept. 118 (1985) 1 [inSPIRE].

[8] R.D. Jordan, Effective Field Equations for Expectation Values, Phys. Rev. D 33 (1986) 444 [INSPIRE].

[9] E. Calzetta and B.L. Hu, Closed Time Path Functional Formalism in Curved Space-Time: Application to Cosmological Back Reaction Problems, Phys. Rev. D 35 (1987) 495 [InSPIRE].

[10] L.H. Ford and R.P. Woodard, Stress tensor correlators in the Schwinger-Keldysh formalism, Class. Quant. Grav. 22 (2005) 1637 [gr-qc/0411003] [INSPIRE].

[11] K.E. Leonard and R.P. Woodard, Graviton Corrections to Maxwell's Equations, Phys. Rev. D 85 (2012) 104048 [arXiv:1202.5800] [INSPIRE].

[12] J.F. Donoghue, Leading quantum correction to the Newtonian potential, Phys. Rev. Lett. 72 (1994) 2996 [gr-qc/9310024] [INSPIRE].

[13] J.F. Donoghue, General relativity as an effective field theory: The leading quantum corrections, Phys. Rev. D 50 (1994) 3874 [gr-qc/9405057] [INSPIRE]. 
[14] N.E.J. Bjerrum-Bohr, J.F. Donoghue and B.R. Holstein, Quantum corrections to the Schwarzschild and Kerr metrics, Phys. Rev. D 68 (2003) 084005 [Erratum ibid. 71 (2005) 069904] [hep-th/0211071] [INSPIRE].

[15] N.E.J. Bjerrum-Bohr, J.F. Donoghue and B.R. Holstein, Quantum gravitational corrections to the nonrelativistic scattering potential of two masses, Phys. Rev. D 67 (2003) 084033 [Erratum ibid. 71 (2005) 069903] [hep-th/0211072] [INSPIRE].

[16] N.E.J. Bjerrum-Bohr, Leading quantum gravitational corrections to scalar QED, Phys. Rev. D 66 (2002) 084023 [hep-th/0206236] [INSPIRE].

[17] S.P. Miao, T. Prokopec and R.P. Woodard, Deducing Cosmological Observables from the S-matrix, Phys. Rev. D 96 (2017) 104029 [arXiv:1708.06239] [INSPIRE].

[18] J.F. Donoghue and T. Torma, On the power counting of loop diagrams in general relativity, Phys. Rev. D 54 (1996) 4963 [hep-th/9602121] [INSPIRE].

[19] S. Deser, General Relativity and the Divergence Problem in Quantum Field Theory, Rev. Mod. Phys. 29 (1957) 417 [InSPIRE].

[20] B.S. DeWitt and R.W. Brehme, Radiation damping in a gravitational field, Annals Phys. 9 (1960) 220 [INSPIRE].

[21] N.C. Tsamis and R.P. Woodard, The Structure of perturbative quantum gravity on a de Sitter background, Commun. Math. Phys. 162 (1994) 217 [INSPIRE].

[22] R.P. Woodard, de Sitter breaking in field theory, in Deserfest: A Celebration of the Life and Works of Stanley Deser, pp. 339-351 (2004) [gr-qc/0408002] [INSPIRE].

[23] K.E. Leonard and R.P. Woodard, Graviton Corrections to Vacuum Polarization during Inflation, Class. Quant. Grav. 31 (2014) 015010 [arXiv: 1304.7265] [INSPIRE].

[24] D. Glavan, S.P. Miao, T. Prokopec and R.P. Woodard, Electrodynamic Effects of Inflationary Gravitons, Class. Quant. Grav. 31 (2014) 175002 [arXiv:1308.3453] [INSPIRE].

[25] C.L. Wang and R.P. Woodard, Excitation of Photons by Inflationary Gravitons, Phys. Rev. D 91 (2015) 124054 [arXiv: 1408.1448] [INSPIRE].

[26] D. Glavan, S.P. Miao, T. Prokopec and R.P. Woodard, Graviton Loop Corrections to Vacuum Polarization in de Sitter in a General Covariant Gauge, Class. Quant. Grav. 32 (2015) 195014 [arXiv:1504.00894] [INSPIRE].

[27] D. Glavan, S.P. Miao, T. Prokopec and R.P. Woodard, One loop graviton corrections to dynamical photons in de Sitter, Class. Quant. Grav. 34 (2017) 085002 [arXiv:1609.00386] [INSPIRE].

[28] D. Glavan, S.P. Miao, T. Prokopec and R.P. Woodard, Graviton Propagator in a 2-Parameter Family of de Sitter Breaking Gauges, JHEP 10 (2019) 096 [arXiv:1908.06064] [InSPIRE]. 